FORSCHUNGSZENTRUM ROSSENDORF e.v.

FZR 93 - 26

November 1993

A. Pfitzner, W. Cassing and A. Peter

Vibrations versus collisions and the iterative structure of two-body dynamics 


\title{
Vibrations versus collisions and the iterative structure of two-body dynamics
}

\author{
A. Pfitzner, W. Cassing ${ }^{1}$ and A. Peter ${ }^{1}$ \\ Forschungszentrum Rossendorf, Institut für Kern- und Hadronenphysik \\ 01314 Dresden \\ ${ }^{1}$ Universität Giessen, Institut für theoretische Physik \\ 35392 Giessen
}

November 26, 1993

\begin{abstract}
The two-body correlation function is decomposed into two channel correlation functions for the pp- and the ph-channel. The associated coupled equations describe the evolution in the respective channels as well as their mixing. Integration of the phchannel in terms of vibrational RPA-states yields a closed equation for the correlations in the pp-channel comprising phonon-particle coupling and a memory term.In the stationary limit the equation for a generalised effective interaction is derived which iterates both the G-matrix (ladders) and the polarisation matrix (loops), thus accounting nonperturbatively for the mixing of ladders and loops.
\end{abstract}

\section{Introduction}

A still outstanding problem in two-body dynamics concerns the simultaneous nonperturbative consideration of pp- (hh-) and ph-interactions, i.e. the consistent integration of both the pp- and the ph-channel. As numerically tested in [1] within the concept of correlation dynamics [2]-[4] the short-range part of the two-body interaction produces correlations mainly in the pp-channel, while the long-range part favours the ph-channel. These results are in agreement with earlier discussions by Abrikosov et. al. [5] for infinite matter and by Migdal [6] for finite nuclei which show that the sum of ladder diagrams should be essential at large momentum transfer whereas one expects loop diagrams to be dominant at small momentum transfer. In this sense, the simultaneous consideration of both channels corresponds to a consistent treatment of short- and long-range correlations (including their mixing), and therefore of collisional and collective aspects in nuclear dynamics.

Because a numerical solution of the equation for the two-body correlations is available at most for light nuclei and in a restricted single-particle basis it is of current interest to make the iterative structure of this equation more transparent, in particular with respect to the mutual influence of short- and long-range correlations. This should help for a better understanding of the interplay between collisions and vibrations during the evolution. Formally, this mutual influence shows up in mixed diagrams combining ladders and loops. The importance of this mixing for the evaluation of occupation number distributions and of damping widths for single-particle and collective excitations is demonstrated in [1] within a model appropriate for light nuclei. 


\section{Channel correlations and the retarded two-body equation}

The nuclear many-body problem on the two-body level can be formulated in terms of coupled equations of motion for the one-body density $\rho$ and the two-body correlation function $C$ [3], which read in short-hand notation

$$
\begin{aligned}
i \dot{\rho} & =[h, \rho]+\operatorname{tr}_{(2)}[v, C] \\
i \dot{C} & =[h(1)+h(2), C]+\left[v^{=}, \rho_{20}+C\right]+\mathcal{A}(1+\mathcal{P} \mathcal{P}) v^{\perp} C .
\end{aligned}
$$

Here we have used the bare mean field $h=\hat{t}+\operatorname{tr} v^{a} \rho$, where $\hat{t}$ is the kinetic energy operator, and superscript $a$ means antisymmetrisation. Further, $\rho_{20}=\mathcal{A} \rho \rho$ is the uncorrelated two-body density, and $\mathcal{A}$ and $\mathcal{P}$ denote the antisymmetrisation and permutation operator, respectively. The in-medium interactions $v^{=}=Q^{=} v$ and $v^{\perp}=Q^{\perp} v^{a}$ are density-dependent via the blocking operators which read in an arbitrary single-particle basis $Q_{\alpha \beta \alpha^{\prime} \beta^{\prime}}^{=}=\delta_{\alpha \alpha^{\prime}} \delta_{\beta \beta^{\prime}}-\delta_{\alpha \alpha^{\prime}} \rho_{\beta \beta^{\prime}}-\delta_{\beta \beta^{\prime}} \rho_{\alpha \alpha^{\prime}}$ and $Q_{\alpha \beta \alpha^{\prime} \beta^{\prime}}^{1}=\delta_{\alpha \beta^{\prime}} \rho_{\beta \alpha^{\prime}}-\delta_{\beta \alpha^{\prime}} \rho_{\alpha \beta^{\prime}}$ (c.f.[7]). In a basis that diagonalises $\rho$ one verifies that $Q^{=}$projects on pp- and hh- states in the HF-limit while $Q^{\perp}$ projects on $\mathrm{ph}$ - and hp- states.

If we subdivide the correlation function $C$ into channel correlations according to

$$
C=C^{=}+C^{\perp}
$$

the channel correlations follow the coupled set of equations

$$
\begin{aligned}
i \dot{C}^{=}= & {\left[H_{0}^{=}, C^{=}\right]+\left[v^{=}, C^{=}+C^{\perp}+\rho_{20}\right] } \\
i \dot{C}^{\perp}= & (1+\mathcal{P} \mathcal{P}) H_{0}^{\perp} C^{\perp}+ \\
& +\mathcal{A}(1+\mathcal{P} \mathcal{P}) v^{\perp}\left(C^{\perp}+C^{=}\right)
\end{aligned}
$$

or, in an arbitrary single-particle basis,

$$
\begin{aligned}
i \dot{C}_{\alpha \beta \alpha^{\prime} \beta^{\prime}}^{=}= & \left\langle\alpha, \beta \|\left[H_{0}^{-}, C^{=}\right] \mid \alpha^{\prime}, \beta^{\prime}\right\rangle+\left\langle\alpha, \beta \|\left[v^{=}, C^{=}+C^{\perp}+\rho_{20}\right] \mid \alpha^{\prime}, \beta^{\prime}\right\rangle \\
i \dot{C}_{\alpha \beta \alpha^{\prime} \beta^{\prime}}^{\perp}= & \left(1+\mathcal{P}_{\alpha \beta} \mathcal{P}_{\alpha^{\prime} \beta^{\prime}}\right) \sum_{\gamma \gamma^{\prime}} H_{\alpha \gamma^{\prime} \alpha^{\prime} \gamma}^{0 \perp} C_{\gamma \beta \gamma^{\prime} \beta^{\prime}}^{\perp}+ \\
& +\mathcal{A}_{\alpha \beta}\left(1+\mathcal{P}_{\alpha \beta} \mathcal{P}_{\alpha^{\prime} \beta^{\prime}}\right) \sum_{\gamma \gamma^{\prime}} v_{\alpha \gamma^{\prime} \alpha^{\prime} \gamma}^{\perp}\left(C_{\beta \gamma \beta^{\prime} \gamma^{\prime}}^{\perp}+C_{\beta \gamma \beta^{\prime} \gamma^{\prime}}^{=}\right) .
\end{aligned}
$$

Observing that the mean-field contributions in (6) and (7) with

$$
\begin{aligned}
\left\langle\alpha \beta\left|H_{0}^{=}\right| \gamma \gamma^{\prime}\right\rangle & =h_{\alpha \gamma} \delta_{\beta \gamma^{\prime}}+\delta_{\alpha \gamma} h_{\beta \gamma^{\prime}} \\
H_{\alpha \gamma^{\prime} \alpha^{\prime} \gamma}^{0 \perp} & =h_{\alpha \gamma} \delta_{\gamma^{\prime} \alpha^{\prime}}-\delta_{\alpha \gamma} h_{\gamma^{\prime} \alpha^{\prime}}
\end{aligned}
$$

may be written either in "horizontal" or "vertical" form one easily proves that the sum of (6) and (7) reproduces the original equation (2) for $C_{\alpha \beta \alpha^{\prime} \beta^{\prime}}$. Eqs. (6) and (7) describe the evolution of correlations in the $\mathrm{pp}$ - and $\mathrm{ph}$-channel, respectively. Their mutual coupling is accounted for by the inhomogeneous terms which comprise $C^{\perp}$ in the pp- and $C^{=}$in the ph-channel. To facilitate the following discussion, we adopt a single-particle basis with $h_{\alpha \gamma}=\delta_{\alpha \gamma} \epsilon_{\alpha \varepsilon}$ and $\rho_{\alpha \gamma} \approx \delta_{\alpha \gamma} n_{\alpha}$. Further we assume the occupation numbers $n_{\alpha}$ to be time-independent which means a decoupling from (1), and neglect the exchange term in the ph-channel dropping $\mathcal{A}_{\alpha \beta}$ in (7). Within these assumptions (7) reads in short-hand notation

$$
i \dot{C}^{\perp}=(1+\mathcal{P} \mathcal{P}) H^{\perp} C^{\perp}+(1+\mathcal{P} \mathcal{P}) v^{\perp} C^{=}
$$


with the RPA-hamiltonian $H^{\perp}=H_{0}^{\perp}+v^{\perp}$ and its matrix elements in the adopted singleparticle basis

$$
H_{\alpha \gamma^{\prime} \alpha^{\prime} \gamma}^{\perp}=\left(\epsilon_{\alpha}-\epsilon_{\alpha^{\prime}}\right) \delta_{\alpha \gamma} \delta_{\alpha^{\prime} \gamma^{\prime}}+\left(n_{\alpha^{\prime}}-n_{\alpha}\right) v_{\alpha \gamma^{\prime} \alpha^{\prime} \gamma}^{a}
$$

For an integration of (10) it suggests itself to transform this equation into the RPA-basis. The RPA-eigenstates $\chi^{\mu}$ and $\chi^{\mu *}$ are given by the secular equations

$$
\begin{aligned}
& \sum_{\gamma \gamma^{\prime}} H_{\alpha \gamma^{\prime} \alpha^{\prime} \gamma}^{\perp} \chi_{\gamma \gamma^{\prime}}^{\mu}=\Omega_{\mu} \chi_{\alpha \alpha^{\prime}}^{\mu} \\
& \sum_{\gamma \gamma^{\prime}} \chi_{\gamma \gamma^{\prime}}^{\mu *} H_{\gamma^{\prime} \beta \gamma \beta^{\prime}}^{\perp \dagger}=\Omega_{\mu} \chi_{\beta \beta^{\prime}}^{\mu *}
\end{aligned}
$$

The matrix elements of the hermitean conjugate operator $H^{\perp \dagger}$ are related to those of $H^{\perp}$ by

$$
H_{\gamma^{\prime} \beta \gamma \beta^{\prime}}^{\perp}=-H_{\beta \gamma^{\prime} \beta^{\prime} \gamma}^{\perp}
$$

Making use of the orthogonality and completeness relation(c.f.[8])

$$
\begin{aligned}
\sum_{\alpha \alpha^{\prime}} \tilde{\chi}_{\alpha \alpha^{\prime}}^{\mu *} \chi_{\alpha \alpha^{\prime}}^{\nu} & =\delta_{\mu \nu} N_{\mu} \\
\sum_{\mu} N_{\mu} \chi_{\alpha \alpha^{\prime}}^{\mu} \chi_{\beta \beta^{\prime}}^{\mu *} & =\delta_{\alpha \beta} \delta_{\alpha^{\prime} \beta^{\prime}}\left(n_{\alpha^{\prime}}-n_{\alpha}\right)
\end{aligned}
$$

the relations between matrix elements in the single-particle- and the RPA-basis read

$$
\begin{aligned}
C_{\alpha \beta \alpha^{\prime} \beta^{\prime}}^{\perp} & =\sum_{\mu \nu} N_{\mu} N_{\nu} \chi_{\alpha \alpha^{\prime}}^{\mu} \chi_{\beta \beta^{\prime}}^{\nu *} C_{\mu \nu} \\
C_{\mu \nu} & =\sum_{\alpha \alpha^{\prime} \beta \beta^{\prime}} \tilde{\chi}_{\alpha \alpha^{\prime}}^{\mu *} \tilde{\chi}_{\beta \beta^{\prime}}^{\nu} C_{\alpha \beta \alpha^{\prime} \beta^{\prime}}^{\perp},
\end{aligned}
$$

with $N_{\mu}=1(-1)$ for $\mu>0(<0)$ and $\tilde{\chi}_{\alpha \alpha^{\prime}}^{\mu}=\left(n_{\alpha^{\prime}}-n_{\alpha}\right)^{-1} \chi_{\alpha \alpha^{\prime}}^{\mu}$. The equation for $C_{\mu \nu}$ follows from (18) using (10)

$$
\begin{aligned}
i \dot{C}_{\mu \nu} & =\left(\Omega_{\mu}-\Omega_{\nu}\right) C_{\mu \nu}+\sum_{\gamma \gamma^{\prime} \lambda \lambda^{\prime}} K_{\gamma \lambda \gamma^{\prime} \lambda^{\prime}}^{\mu \nu} C_{\gamma \lambda \gamma^{\prime} \lambda^{\prime}}^{=} \\
K_{\gamma \lambda \gamma^{\prime} \lambda^{\prime}}^{\mu \nu} & =\tilde{\chi}_{\lambda \lambda^{\prime}}^{\nu} \theta_{\gamma \gamma^{\prime}}^{\mu *}+\tilde{\chi}_{\gamma \gamma^{\prime}}^{\mu *} \theta_{\lambda \lambda^{\prime}}^{-\nu},
\end{aligned}
$$

where we have introduced the phonon-particle vertices $\left(\Omega_{-\nu}=-\Omega_{\nu}\right)$

$$
\begin{aligned}
& \theta_{\gamma \gamma^{\prime}}^{\mu}:=\sum_{\alpha \alpha^{\prime}} v_{\gamma \alpha^{\prime} \gamma^{\prime} \alpha}^{a} \chi_{\alpha \alpha^{\prime}}^{\mu}=\left(\Omega_{\mu}-\epsilon_{\gamma}+\epsilon_{\gamma^{\prime}}\right) \tilde{\chi}_{\gamma \gamma^{\prime}}^{\mu \mu} \\
& \theta_{\lambda \alpha^{\prime}}^{-\nu}:=\sum_{\beta \beta^{\prime}} \chi_{\beta \beta^{\prime}}^{\nu} v_{\lambda^{\prime} \beta \lambda \beta \beta^{\prime}}^{a}=\left(\Omega_{-\nu}-\epsilon_{\lambda}+\epsilon_{\lambda^{\prime}}\right) \tilde{\chi}_{\lambda \lambda^{\prime \prime}}^{\nu}
\end{aligned}
$$

These relations may be proved using the RPA-equations (12) and (13). After integration of (19) the resulting solution $C_{\mu \nu}(t)$ is transformed back into the single-particle basis yielding $\left(\Omega_{\mu \nu}=\Omega_{\mu}-\Omega_{\nu}\right)$

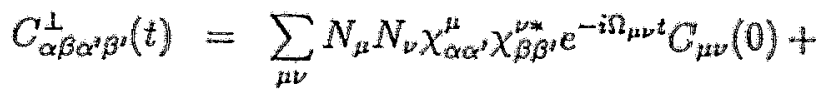

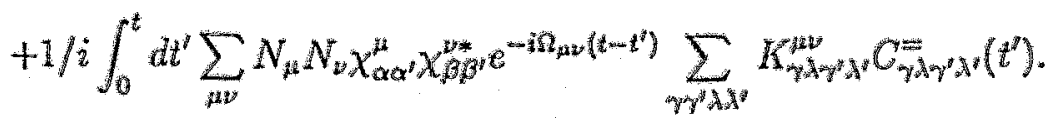


The first term is a superposition of oscillations around the initial values $C_{\mu \nu}(0)$ and represents a solution of the homogeneous equation that follows when dropping the term with $C^{=}$ in (10). The second term comprises the coupling with the pp-channel.

Insertion of (23) into (6) leads to a retarded two-body equation in the pp-channel,

$$
\begin{aligned}
i \dot{C}_{\alpha \beta \alpha^{\prime} \beta^{\prime}}^{=}= & <\alpha \beta\left|\left[H^{=}, C^{=}\right]\right| \alpha^{\prime} \beta^{\prime}>+<\alpha \beta\left|\left[v^{=}, \rho_{20}+C^{\perp 0}\right]\right| \alpha^{\prime} \beta^{\prime}>+ \\
& +\int_{0}^{t} d t^{\prime} \sum_{\gamma \lambda \gamma^{\prime} \lambda^{\prime}}<\alpha \beta\left|M^{\gamma \lambda \gamma^{\prime} \lambda^{\prime}}\left(t-t^{\prime}\right)\right| \alpha^{\prime} \beta^{\prime}>C_{\gamma \lambda \gamma^{\prime} \lambda^{\prime}}^{=}\left(t^{\prime}\right)
\end{aligned}
$$

with $H^{=}=H_{0}^{=}+v^{=}$, the memory-kernel

$$
M^{\gamma \lambda \gamma^{\prime} \lambda^{\prime}}\left(t-t^{\prime}\right)=\sum_{\mu \nu} N_{\mu} N_{\nu} K_{\gamma \lambda \gamma^{\prime} \lambda^{\prime}}^{\mu \nu}\left[v^{=}, 1 / i \chi^{\mu} \circ \chi^{\nu *} e^{-i \Omega_{\mu \nu}\left(t-t^{\prime}\right)}\right]
$$

and the initial correlations

$$
C^{\perp 0}=\sum_{\mu \nu} N_{\mu} N_{\nu} C_{\mu \nu}(0) \chi^{\mu} \circ \chi^{\nu *} e^{-i \Omega_{\mu \nu} t}
$$

Here, we used the notations $[A, B]=A B-B^{\dagger} A^{\dagger}$ and $(A \circ B)_{1234}=A_{13} B_{24}$. The coupling with the ph-channel produces two contributions in the pp-channel: (1) A source term originating from the initial correlations $C_{\mu \nu}(0)$ in the ph-channel, and (2) a retardation term comprising memory effects due to phonon-particle coupling. The non-locality in time of the memory-kernel - characterised by a memory-time $\tau^{*}$ - is controlled by the number of collective states contributing to the sum $\sum_{\mu \nu}$ in (21). On the other hand, the change in time of $C^{=}\left(t^{\prime}\right)$ - characterised by a relaxation time $\tau_{\text {rel }}$ - is determined by the strength of the interaction. Only for $\tau^{*} \ll \tau_{\text {rel }}$ one can replace t' by $\mathrm{t}$ in $C^{=}\left(t^{\prime}\right)$ and extend the integration to infinity (Markov-limit).

The channel-mixing is described by the retardation term in (24). In order to allow for a separate treatment of this mixing we introduce a corresponding mixing correlator $\triangle C$ by $C^{=}=c^{=}+\Delta C$, with

$$
i \dot{c}^{=}=\left[H^{=}, c^{=}\right]+\left[v^{=}, \rho_{20}+C^{\perp 0}\right] .
$$

Without $C^{\perp 0}(t)$ eq.(27) accounts for a resummation of ladders as known from time-dependent G-matrix theory [9]. The additional driving term $\left[v^{=}, C^{\perp 0}\right]$ modifies this by coupling with oscillations due to the initial correlations in the ph-channel. Now, from (24) we obtain for the correlations due to a mixing of ladders and loops

$$
i \Delta C=\left[H^{=}, \Delta C\right]+\int_{0}^{t} d t^{\prime} \sum_{\gamma \lambda \gamma^{\prime} \lambda^{\prime}} M^{\gamma \lambda \gamma^{\prime} \lambda^{\prime}}\left(t-t^{\prime}\right)\left(\Delta C\left(t^{\prime}\right)+c^{=}\left(t^{\prime}\right)\right)_{\gamma \lambda \gamma^{\prime} \lambda^{\prime}}
$$

In order to study the iterative structure it is more convenient to use the equivalent integral equation

$$
\Delta C(t)=\Delta c^{=}(t)+\int_{0}^{t} d t^{\prime} \sum_{121^{\prime} 2^{\prime}} W^{121^{\prime} 2^{\prime}}\left(t-t^{\prime}\right) \Delta C_{121^{\prime} 2^{\prime}}\left(t^{\prime}\right)
$$

with

$$
\Delta c=(t)=\int_{0}^{t} d t^{\prime} \sum_{121^{\prime} 2^{\prime}} W^{121^{\prime} 2^{\prime}}\left(t-t^{\prime}\right) c_{121^{\prime} 2^{\prime}}^{=}\left(t^{\prime}\right)
$$

The integral kernel $W\left(t-t^{\prime}\right)$ may be cast into the form

$$
W^{121^{\prime} 2^{\prime}}\left(t-t^{\prime}\right)=1 / i \int_{0}^{t-t^{\prime}} d \tau e^{-i H^{\prime}=\tau} M^{121^{\prime} 2^{\prime}}\left(t-t^{\prime}-\tau\right) e^{i H^{-\dagger^{\dagger}} \tau}
$$


It is of first order in the phonon-particle vertices (c.f.(25)), but of infinite order in the blocked interaction $v^{=}$. In deriving (30) and (31) we have used the identity $\int_{0}^{t} d t^{\prime} \int_{0}^{t^{\prime}} d t^{\prime \prime}=$ $\int_{0}^{t} d t^{\prime \prime} \int_{t^{\prime \prime}}^{t} d t^{\prime}$. Applying this relation repeatedly we may arrange the solution of (29) in powers of $W$,

$$
\begin{array}{r}
\Delta C_{\alpha \beta \alpha^{\prime} \beta^{\prime}}(t)=\Delta c_{\alpha \beta \alpha^{\prime} \beta^{\prime}}^{=}(t)+\int_{0}^{t} d t^{\prime} \sum_{121^{\prime} 2^{\prime}}\left[W\left(t-t^{\prime}\right)\right. \\
+\int_{t^{\prime}}^{t} d \tau_{1} W\left(t-\tau_{1}\right) W\left(\tau_{1}-t^{\prime}\right)+\int_{t^{\prime}}^{t} d \tau_{1} \int_{t^{\prime}}^{\tau_{1}} d \tau_{2} \\
\left.W\left(t-\tau_{1}\right) W\left(\tau_{1}-\tau_{2}\right) W\left(\tau_{2}-t^{\prime}\right)+\cdots\right]_{\alpha \beta \alpha^{\prime} \beta^{\prime}}^{121^{\prime}} \Delta c_{121^{\prime} 2^{\prime}}^{=}\left(t^{\prime}\right) .
\end{array}
$$

Products of $W$ are formed according to

$$
(W W)_{\alpha \beta \alpha^{\prime} \beta^{\prime}}^{121^{\prime} 2^{\prime}}=\sum_{343^{\prime} 4^{\prime}} W_{\alpha \beta \alpha^{\prime} \beta^{\prime}}^{343^{\prime} 4^{\prime}} W_{343^{\prime} 4^{\prime}}^{121^{\prime} 2^{\prime}}
$$

The solution (32) makes the iterative structure transparent: the resummation of ladders together with a coupling to vibrations contained in each $W$ is followed by a resummation of $W$ and thus of phonon-particle vertices.

Combining all relations with the solution of (27) we are able within our approximations to trace back the total two-body correlations $C$ to the uncorrelated two-body density $\rho_{20}$ and the initial (vibrational) correlations $C^{\perp 0}$ in the ph-channel.

\section{Stationary limit and effective interactions}

In the stationary limit $\dot{C}=0$, two-body dynamics can be traced back to integral equations for frequency-dependent effective interactions. We meet the stationary limit imposing $\dot{C}^{=}=$ 0 and $\dot{C}^{\perp}=0$ in (6) and (7), respectively. Strictly speaking, due to the exchange term equation (7) splits up into two equations for the ph-channels $\left(\alpha, \alpha^{\prime}\right)$ and $\left(\alpha, \beta^{\prime}\right)$. Since, however, the equation for channel $\left(\alpha, \beta^{\prime}\right)$ does not provide new aspects with respect to channel mixing it is neglected in the following by dropping $\mathcal{A}_{\alpha \beta}$ in (7). The price for this simplification is the loss of antisymmetry with respect to the labels $(\alpha, \beta)$ and $\left(\alpha^{\prime} \beta^{\prime}\right)$ in the ph-channel. Considerations with three coupled equations are made e.g. in [10] using Green-function techniques.

Equations (6) and (7) are solved in the stationary limit by the correlations $C^{=0}$ and $C^{\perp 0}$ which can be determined from the two coupled equations

$$
\begin{aligned}
\left(\omega^{=}-\epsilon_{\alpha}-\epsilon_{\beta}\right) C_{\alpha \beta \alpha^{\prime} \beta^{\prime}}^{=0} & =<\alpha \beta\left|v^{=}\left(C^{=0}+C^{\perp 0}+\rho_{20}^{0}\right)\right| \alpha^{\prime} \beta^{\prime}> \\
\left(\omega^{\perp}-\epsilon_{\alpha}+\epsilon_{\alpha^{\prime}}\right) C_{\alpha \beta \alpha^{\prime} \beta^{\prime}}^{\perp 0} & =\sum_{\gamma \gamma^{\prime}} v_{\alpha \gamma^{\prime} \alpha^{\prime} \gamma}^{\perp}\left(C^{\perp 0}+C^{=0}\right)_{\gamma \beta \gamma^{\prime} \beta^{\prime}},
\end{aligned}
$$

where $\rho_{20}$ is the stationary uncorrelated two-body-density. This can be seen by subtracting from (34) and (35) the respective hermitean conjugate equation. By introducing effective interactions $G\left(\omega^{=}\right)$and $\Pi\left(\omega^{\perp}\right)$ by the definitions

$$
\begin{aligned}
\left.<\alpha \beta\left|v\left(C^{=0}+C^{\perp 0}+\rho_{20}^{0}\right)\right| \alpha^{\prime} \beta^{\prime}\right\rangle & \left.=<\alpha \beta\left|G\left(C^{10}+\rho_{20}^{0}\right)\right| \alpha^{\prime} \beta^{\prime}\right\rangle \\
\sum_{\gamma \gamma^{\prime}} v_{\alpha \gamma^{\prime} \alpha^{\prime} \gamma}^{a}\left(C^{\perp 0}+C^{=0}\right)_{\gamma \beta \gamma^{\prime} \beta^{\prime}} & =\sum_{\gamma \gamma^{\prime}} \Pi_{\alpha \gamma^{\prime} \alpha^{\prime} \gamma} C_{\gamma \beta \gamma^{\prime} \beta^{\prime}}^{=0}
\end{aligned}
$$

and insertion of (36) and (37) into (34) and (35), respectively, we obtain integral equations for the G-matrix and the polarisation matrix $\Pi$ (c.f. also [1])

$$
\begin{aligned}
& G_{\alpha \beta \alpha^{\prime} \beta^{\prime}}=v_{\alpha \beta \alpha^{\prime} \beta^{\prime}}+<\alpha \beta\left|v\left(\omega^{=(+)}-H_{0}^{=}\right)^{-1} Q^{=} G\right| \alpha^{\prime} \beta^{\prime}> \\
& \Pi_{\alpha \beta \beta \alpha^{\prime} \beta^{\prime}}=v_{\alpha \beta \alpha^{\prime} \beta^{\prime}}+\sum_{\gamma \gamma^{\prime}}\left[v^{a}\left(\omega^{\perp(+)}-H_{0}^{\perp}\right)^{-1} Q^{\perp}\right]_{\alpha \gamma^{\prime} \alpha^{\prime} \gamma^{\prime}} \Pi_{\gamma \beta \gamma^{\prime} \beta^{\prime}}
\end{aligned}
$$


with $\omega^{(+)}=\omega+i \eta, \eta \rightarrow 0^{(+)}$. Assuming again $\rho^{0}$ and $h\left(\rho^{0}\right)$ to be diagonal, the matrix elements of the blocking operators simplify to $Q_{\overline{\lambda \gamma^{\prime} \lambda^{\prime} \gamma}}^{=}=\delta_{\lambda \lambda^{\prime}} \delta_{\gamma \gamma^{\prime}}\left(1-n_{\lambda}-n_{\gamma^{\prime}}\right)$ and $Q_{\lambda \gamma^{\prime} \lambda^{\prime} \gamma}^{1}=$ $\delta_{\lambda \gamma} \delta_{\lambda^{\prime} \gamma^{\prime}}\left(n_{\lambda^{\prime}}-n_{\lambda}\right)$, and the mean-field two-body propagators $g^{p p}\left(\omega^{=}\right)=\left[\omega^{=(+)}-H_{0}^{=}\right]^{-1}$ and $g^{p h}\left(\omega^{\perp}\right)=\left[\omega^{\perp(+)}-H_{0}^{\perp}\right]^{-1}$ reduce to

$$
\begin{aligned}
& g_{\alpha \beta \alpha^{\prime} \beta^{\prime}}^{p p}=\delta_{\alpha \alpha^{\prime}} \delta_{\beta \beta^{\prime}}\left[\omega^{(+)}-\epsilon_{\alpha}-\epsilon_{\beta}\right]^{-1} \\
& g_{\alpha \beta \alpha^{\prime} \beta^{\prime}}^{p h}=\delta_{\alpha \beta^{\prime}} \delta_{\beta \alpha^{\prime}}\left[\omega^{\perp(+)}-\epsilon_{\alpha}+\epsilon_{\alpha^{\prime}}\right]^{-1} .
\end{aligned}
$$

A representation of (38) and (39) in terms of diagrams is given in fig.1 and fig.2, respectively. The iterative structure makes explicit that $G$ accounts for a resummation of ladders, while $\Pi$ accounts for a resummation of loops.

Equations (38) and (39) describe the stationary limit in the absence of channel mixing, i.e. when dropping $C^{\perp 0}$ in (34) and $C^{=0}$ in (35). In order to account for the mutual influence of both channels it is tempting to construct a total G-matrix $G^{\text {tot }}\left(\omega^{=}, \omega^{\perp}\right)$ in terms of $G\left(\omega^{=}\right)$ and $\Pi\left(\omega^{\perp}\right)$. This can be achieved by the definition

$$
G^{t o t} \rho_{20}^{0}=G\left(C^{\perp 0}+\rho_{20}^{0}\right)
$$

which yields, together with (36) and (34), a relation between $C^{=0}$ and $G^{\text {tot }}$. Furthermore, insertion of (37) into (35) allows to express $C^{\perp 0}$ in terms of $\Pi$ and $C^{=0}$. Finally, combining all relations with (42) one arrives at an equation for $G^{\text {tot }}$ which may be cast into the form

$$
G_{\alpha \beta \alpha^{\prime} \beta^{\prime}}^{t o t}=G_{\alpha \beta \alpha^{\prime} \beta^{\prime}}+\sum_{\lambda \kappa \kappa^{\prime}} \Gamma\left(\omega^{\perp}\right)_{\alpha \beta \kappa^{\prime} \alpha^{\prime} \kappa \lambda} \frac{1-n_{\kappa}-n_{\lambda}}{\omega=(+)-\epsilon_{\kappa}-\epsilon_{\lambda}} G_{\kappa \lambda \kappa^{\prime} \beta^{\prime}}^{\text {tot }}
$$

The frequency-dependent 6 -label interaction $\Gamma\left(\omega^{\perp}\right)$ is a combination of $G, \Pi$ and a phpropagator,

$$
\Gamma\left(\omega^{\perp}\right)_{\alpha \beta \kappa^{\prime} \alpha^{\prime} \kappa \lambda}=\sum_{\gamma} G_{\alpha \beta \gamma \lambda} \frac{n_{\alpha^{\prime}}-n_{\gamma}}{\omega^{\perp(+)}-\epsilon_{\gamma}+\epsilon_{\alpha^{\prime}}} \Pi_{\gamma^{\prime} \alpha^{\prime} \kappa} .
$$

Eq.(43) is not an usual Bethe-Goldstone equation because not two but three labels of $G^{\text {tot }}$ are involved in the summation on the r.h.s.. Its iterative structure is displayed in fig.3. The successive iteration proceeds in powers of $\Gamma$ connected by a respective pp-propagator. A diagram of second order in $\Gamma$ is shown in fig.4.

Equation (43) describes nonperturbatively the channel mixing in the stationary limit of two-body dynamics. For transparency $\rho^{0}$ was assumed to be diagonal. This assumption can easily be dropped replacing $1-n_{\kappa}-n_{\lambda}$ in (43) and $n_{\alpha^{\prime}}-n_{\gamma}$ in (44) by the non-diagonal expressions for $Q^{=}$and $Q^{\perp}$, respectively. For a complete description of a stationary system the equations for the effective interactions must be completed by the stationary one-body equation

$$
0=\left[\hat{t}, \rho^{0}\right]+\operatorname{tr}_{2}\left[v, \rho_{20}^{0}+C^{0}\right]
$$

Here, $\hat{t}$ is the kinetic energy operator, and $t r_{2}$ means the trace over the second particle. With $C^{0}=C^{10}+C^{=0}$ and the definitions (36) and (42) we obtain

$$
0=\left[\hat{i}, \rho^{0}\right]+t r_{2}\left[G^{t o t}, \rho_{20}^{0}\right]
$$

This equation for $\rho^{0}$ together with the equations for $G^{\text {tot }}, G$ and $I$ represent a closed set of equations for the stationary limit of the system. This may be viewed as basis for a more sophisticated treatment of the true (correlated) ground state in the sense that channel mixing and hence the mutual influence of long- and short-range correlations is nonperturbatively included. 


\section{In-medium scattering approach}

Short-range correlations are associated with multiple pp-(or hh-) collisions mediated by the in-medium interaction $v^{=}$. The in-medium scattering approach is based on the assumption that this collisions happen on a time-scale which is short as compared with the time in between the collisions. Neglecting, for a moment, long-range correlations and approximating the "collision-free" evolution on the long time-scale by the uncorrelated two-body density,

$$
i \dot{\rho}_{20}=\left[H_{0}^{=}, \rho_{20}\right]
$$

we obtain for the short-range correlations

$$
C^{=}=-\rho_{02}+\Omega(E) \rho_{20} \Omega(E)^{\dagger}
$$

where the two-body Moeller operator $\Omega(E)$ depends on the total energy of the "scatteringsystem" and follows the equation

$$
\Omega(E)=1+\left[E^{+}-H_{0}^{=}\right]^{-1} v^{=} \Omega(E) .
$$

This has been shown in $[3,9]$ by an approximate integration of the two-body equation. Equation (49) is equivalent to (38) when defining the G-matrix by $G(E)=v \Omega(E)$.

Within this concept of two different time-scales long-range-correlations are included assuming that they are operative essentially on the long time-scale, i.e. in between the collisions. This allows to apply the same integration procedure from $[3,9]$ to eq.(4), which yields

$$
C^{=} \approx-\rho_{20}-C^{\perp}+\Omega\left(\rho_{20}+C^{\perp}\right) \Omega^{\dagger}
$$

The Moeller-operators may be expressed in terms of G,

$$
\Omega(E)=1+g(E) Q^{=} G(E)
$$

with $g(E)=\left[E^{+}-H_{0}^{=}\right]^{-1}$. Insertion of $(50)$ into (10) provides an equation for the long-range correlations

$$
\begin{aligned}
i \dot{C}^{\perp}= & (1+\mathcal{P} \mathcal{P}) H^{\perp} C^{\perp}+(1+\mathcal{P} \mathcal{P}) v^{\perp}\left(\left[g Q^{=} G, C^{\perp}\right]_{+}+\right. \\
& \left.g Q^{=} G C^{\perp} G^{\dagger} Q^{=} \dot{g}^{\dagger}\right)+D\left(\rho_{20}\right) .
\end{aligned}
$$

We observe that channel mixing produces a driving term

$$
D\left(\rho_{20}\right)=(1+\mathcal{P} \mathcal{P}) v^{\perp}\left(\Omega \rho_{20} \Omega^{\dagger}-\rho_{20}\right)
$$

as well as an additional interaction term of mixed structure (second term on the r.h.s.): $C^{\perp}$ is horizontally connected with $\mathrm{G}$ but the resulting expression is vertically connected with $v^{\perp}$. Both terms are non-hermitean due to the propagator $g(E)$.

Equation (52) holds for an arbitrary single-particle basis. Together with (1) and (49) it represents a closed set of equations for the long-range correlations $C^{\perp}$, the $G$-matrix $G$ and the one-body density $\rho$. The one-body equation reads, with (3) and (50),

$$
i \dot{\rho}-[\tilde{h}, \rho]=I_{\text {coll }}(\rho)+\operatorname{tr}_{2}\left[G, C^{\perp}\right]+\operatorname{tr}_{2}\left[G C^{\perp} G^{\dagger}, Q^{-} g^{\dagger}\right]
$$

with the on-shell collision term from time-dependent G-matrix theory

$$
I_{\text {coll }}=i t r_{2}\left(\left[\operatorname{Im} G, \rho_{20}\right]_{4}-\left[G \rho_{20} G^{4}, Q^{=} \operatorname{Im}\right]_{+}\right)
$$


and the renormalized mean field $\tilde{h}=\hat{t}+\operatorname{tr} \operatorname{Re} G^{a} \rho$. Equation (54) was used in [11] to study nuclear damping without channel mixing, i.e. with approximate long-range corralations which follow from (10) when dropping the term with $C^{=}$.

Due to the non-hermitean character of (52) we expect that the mixing with the ppchannel leads to damped vibrations in the ph-channel. To make this more transparent we assume - similar as in chapter 2 -that the one-body density appearing in the interaction terms of (52) is diagonal and time-independent. This allows for a transformation into the RPA-basis defined in $(12,13)$. The result is

$$
i \dot{C}_{\mu \nu}=\Omega_{\mu \nu} C_{\mu \nu}+\sum_{\mu^{\prime} \nu^{\prime}} K_{\mu^{\prime} \nu^{\prime}}^{\mu \nu} C_{\mu^{\prime} \nu^{\prime}}+D_{\mu \nu}\left(\rho_{20}\right)
$$

with the complex coupling matrix

$$
\begin{aligned}
& K_{\mu^{\prime} \nu^{\prime}}^{\mu \nu}=N_{\mu^{\prime}} N_{\nu^{\prime}} \sum_{1234} K_{1234}^{\mu \nu} T_{1234}^{\mu^{\prime} \nu^{\prime}} \\
& T_{1234}^{\mu^{\prime} \nu^{\prime}}=<12\left|\left(\Omega \chi^{\mu^{\prime}} \circ \chi^{\nu^{\prime} *} \Omega^{\dagger}-\chi^{\mu^{\prime}} \circ \chi^{\nu^{\prime *}}\right)\right| 34>
\end{aligned}
$$

and the driving term

$$
D_{\mu \nu}\left(\rho_{20}\right)=\sum_{1234} K_{1234}^{\mu \nu}<12\left|\left(\Omega \rho_{20} \Omega^{\dagger}-\rho_{20}\right)\right| 34>
$$

The contributions to the sums in (57) and (59) factorize into a term $K_{1234}^{\mu \nu}$ which is, according to (20), a linear combination of phonon -particle vertices, multiplied by a term which comprises the short-range correlations in form of Moeller operators $\Omega(E)$. In the limit $\Omega=1$, i.e. without collisions, both coupling matrix and driving term vanish. The source for damping is the imaginary part of the coupling matrix. Using (51) we obtain

$$
\begin{aligned}
\operatorname{Im} K_{\mu \nu}^{\mu^{\prime} \nu^{\prime}}= & -N_{\mu^{\prime}} N_{\nu^{\prime}} \sum_{1234} K_{1234}^{\mu \nu}<12 \mid(1+R) \chi^{\mu^{\prime}} \circ \chi^{\nu^{\prime *}} S^{\dagger}- \\
& -S \chi^{\mu^{\prime}} \circ \chi^{\nu^{\prime *}}\left(1+R^{\dagger}\right) \mid 34>,
\end{aligned}
$$

with

$$
\begin{gathered}
R \approx-\operatorname{Img} Q^{=} \operatorname{Im} G \\
S \approx I m g Q^{=} \operatorname{Re} G .
\end{gathered}
$$

Here we have adopted the on-shell approximation $g(E) \approx i \operatorname{Im} g(E)=-i \pi \delta(E-h(1)-h(2))$.

A further evaluation of (56) is achieved by solving the non-hermitean eigenvalue -problem

$$
\begin{array}{r}
\omega_{\eta} \xi^{\eta}=A \xi^{\eta} \\
\xi^{n \dagger} \omega_{\eta}^{*}=\xi^{n \dagger} A^{\dagger}
\end{array}
$$

with $A_{\mu \nu \mu^{\prime} \nu^{\prime}}=\left(\Omega_{\mu}-\Omega_{\nu}\right) \delta_{\mu \mu^{\prime}} \delta_{\nu \nu^{\prime}}+K_{\mu \nu}^{\mu^{\prime} \nu^{\prime}}$. The eigenstates $\xi_{\mu \nu}^{\eta}, \xi_{\mu \nu}^{\eta \dagger}$ form a biorthogonal system which follows the orthogonality relation

$$
\sum_{\mu \nu} \xi_{\mu \nu}^{\eta *} \xi_{\mu \nu}^{\eta^{\prime}}=\delta_{\eta \eta^{\prime}}
$$

An expansion $C_{\mu \nu}(t)=\sum_{\eta_{i}} C_{\eta}(t) \xi_{\mu \nu}^{\eta}$ yields, together with (56), an equation for the components $C_{\eta}(t)$, the solution of which reads

$$
C_{\eta}(t)=e^{-i \omega_{\eta} t} C_{\eta}(0)+1 / i \int_{0}^{t} d t^{\prime} e^{-i \omega_{\eta}\left(t-t^{\prime}\right)} \sum_{\mu \nu} \xi_{\mu \nu}^{\eta \dagger} D_{\mu \nu}\left(\rho_{20}\left(t^{\prime}\right)\right) .
$$


A transformation back into the single-particle basis provides for the long-range correlations

$$
\begin{aligned}
C_{\alpha \beta \alpha^{\prime} \beta^{\prime}}^{\perp} \approx & \sum_{\eta} f_{\alpha \beta \alpha^{\prime} \beta^{\prime}}^{\eta} e^{-i \omega_{\eta} t} C_{\eta}(0)+1 / i \int_{0}^{t} d t^{\prime} \sum_{\eta, 1234} \\
& f_{\alpha \beta \alpha^{\prime} \beta^{\prime}}^{\eta} K_{1234}^{\eta} e^{-i \omega_{\eta}\left(t-t^{\prime}\right)}\left(\Omega \rho_{20}\left(t^{\prime}\right) \Omega^{\dagger}-\rho_{20}\left(t^{\prime}\right)\right)_{1234}
\end{aligned}
$$

with

$$
\begin{aligned}
f_{1234}^{\eta} & =\sum_{\mu \nu} N_{\mu} N_{\nu} \chi_{13}^{\mu} \chi_{24}^{\nu *} \xi_{\mu \nu}^{\eta} \\
K_{1234}^{\eta} & =\sum_{\mu \nu} \xi_{\mu \nu}^{\eta \dagger} K_{1234}^{\mu \nu} .
\end{aligned}
$$

Expression (67) is our central result. The long-range correlations are superpositions of vibrational modes which are damped due to the coupling with short-range correlations. This collisional damping is accounted for in a way which is non-perturbative (i) with respect to the phonon-particle vertices due to the diagonalisation of $A(63,64)$ and (ii) with respect to the two-body collisions due to the G-matrix appearing in (60). Furthermore, after insertion of (67) into (54), we observe that channel mixing produces retardation effects in the one-body equation.

\section{Summary}

In this paper a reformulation of two-body dynamics is presented in which the equation for the two-body correlation function as obtained from the time-dependent density matrix hierarchy is replaced by two coupled equations for correlations in the pp-and the ph-channel. These correlations are identified with short- and long-range correlations and associated with in-medium collisions and vibrational motion, respectively. Their mutual influence (termed channel-mixing) is analised by means of an integral equation for the corresponding mixing correlations which exhibits the iterative structure of two-body dynamics: After a resummation of ladders accounting for in-medium collisions a resummation of loops in terms of vibrational RPA-modes is performed which accounts for particle-phonon coupling. This way the complete two-body correlation function is traced back to the uncorrelated twobody density and the initial vibrations in the ph-channel. We stress that our approach, at variance to other theories, is non-perturbative also with respect to mixed diagrams, that double-counting is avoided and that no linearisation has been implemented (large-amplitude motion).

In the stationary limit, channel-mixing can be traced back to an effective interaction which combines the usual $G$-matrix with the polarisation matrix. The corresponding integral equation differs from the Bethe-Goldstone equation because three labels instead of two are involved in the summation.

Assuming that short- and long-range correlations evolve on two different time-scales the short-range correlations can be integrated in terms of the $G$-matrix. In this in-medium scattering approach (termed time-dependent $G$-matrix theory when neglecting long-range correlations) channel-mixing shows up in the damping of the vibrational modes which constitute the long-range correlations. In the one-body equation in-medium collisions care for both a renormalisation of the mean field and a collision term, whereas channel-mixing produces retardation effects. 


\section{References}

[1] A.Peter, W.Cassing, I.M.Häuser, A.Pfitzner, Nucl.Phys.A, in press

[2] S.J.Wang, W.Cassing, Ann. of Phys. 159(1985)328

[3] W.Cassing, A.Pfitzner, Z.Phys.A337(1990)175

[4] W.Cassing, A.Pfitzner, Z.Phys.A342(1992)161

[5]A.A.Abrikosov, L.P.Gorkov, I.E.Dzyaloshinski: Quantum Field Theoretical Methods in Statistical Physics, Pergamon, Oxford, 1965

[6] A.B.Migdal: Theory of Finite Fermi Systems and Application to Atomic Nuclei, Wiley Interscience, New York, 1967

[7] W.Cassing, A.Peter, A.Pfitzner, Nucl.Phys.A561(1993)133

[8] S.Adachi, P.Schuck, Nucl.Phys.A496(1989)485

[9] W.Cassing, K.Niita, S.J.Wang, Z.Phys.A331(1988)439

[10] R.Krieg, thesis, Erlangen 1991

[11] A.Pfitzner, W.Cassing, Z.Phys.A, submitted 
Figure captions

Fig.1

Integral equation for the G-matrix<smiles>CC1C(C)C(C)C(C)C1C</smiles><smiles>[CH]</smiles><smiles>CC(C)(C)C</smiles>

Fig.2

Integral equation for the polarisation matrix<smiles>CC1C(C)C(C)C1C</smiles><smiles>[Tl]</smiles><smiles>CC(C)(C)C</smiles>

Fig. 3

Integral equation for $G^{\text {tot}}$; the operators $G$ and $\pi$ in the second diagram on the r.h.s. are connected dy one line forming the 6 -label operator $\Gamma$.

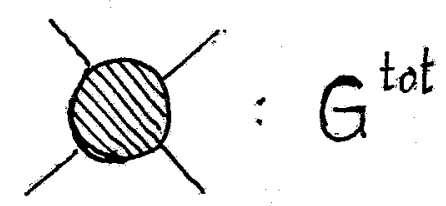

Fig.4

Second-order contribution in $\Gamma$. 

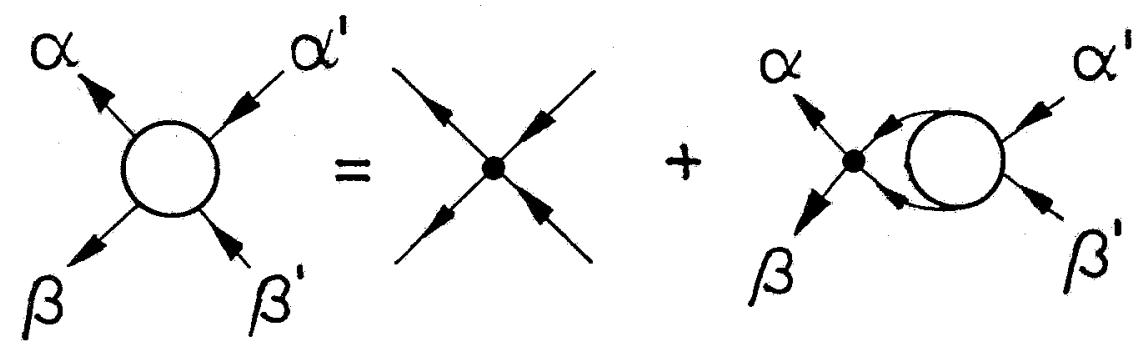

Fig. 1

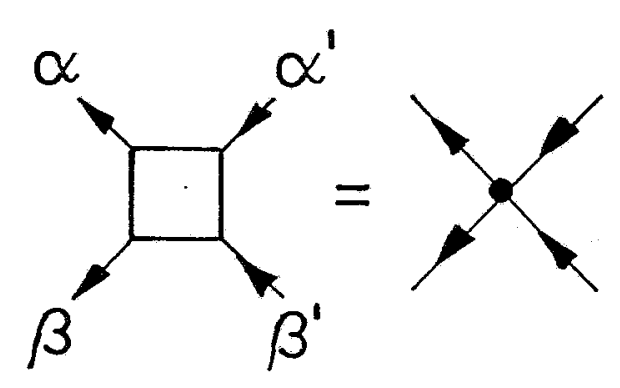

Fig. 2
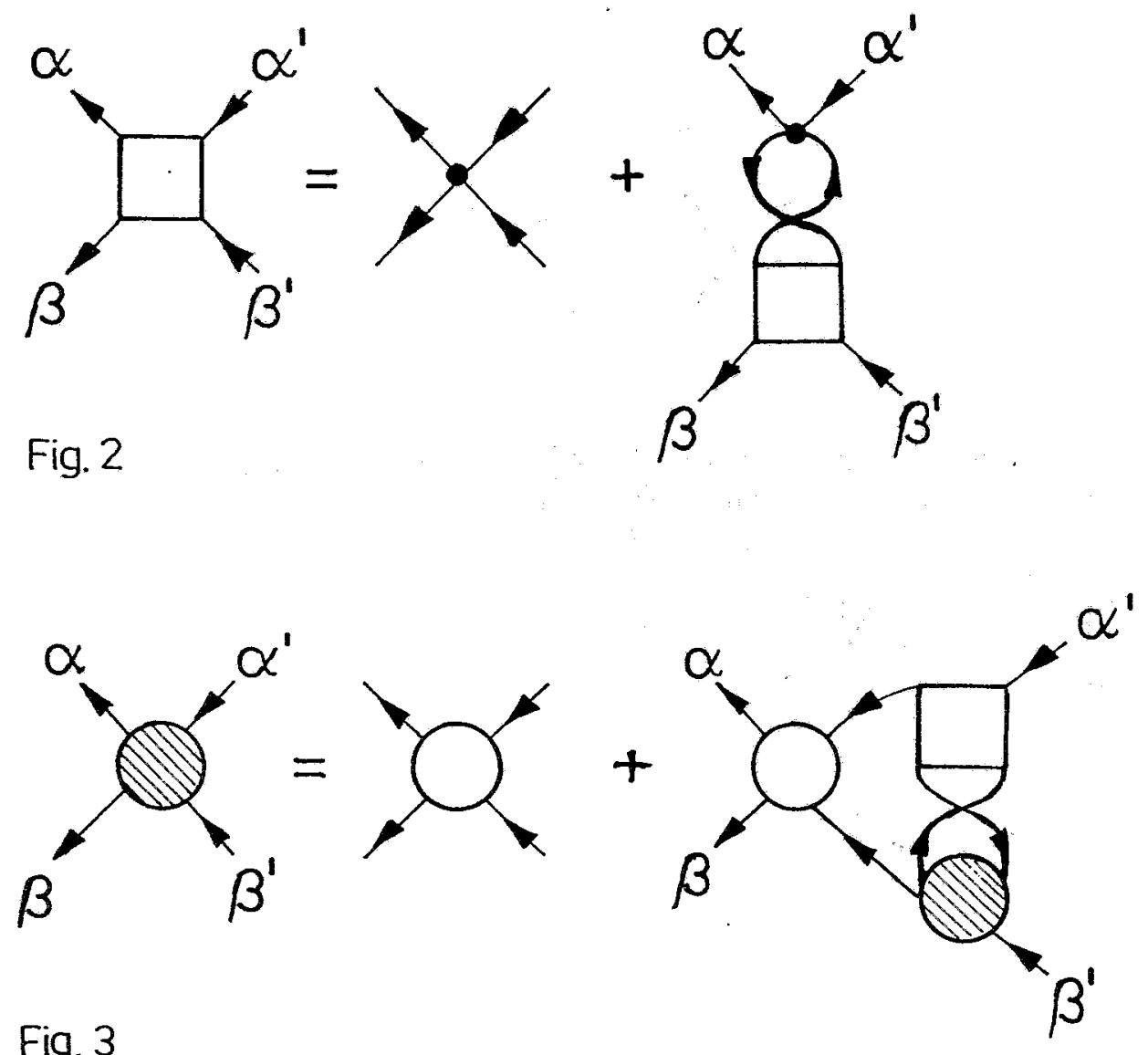

Fig. 3

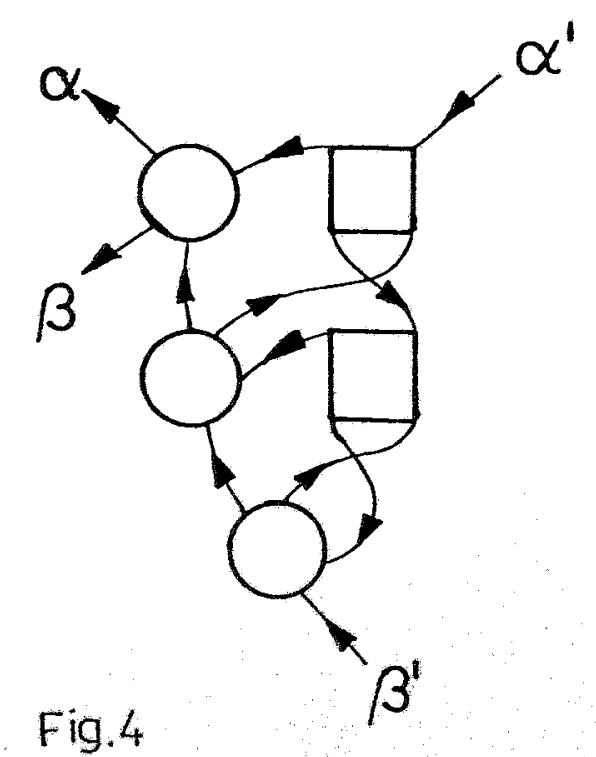

